Research Article

\title{
Combination of Deep Recurrent Neural Networks and Conditional Random Fields for Extracting Adverse Drug Reactions from User Reviews
}

\author{
Elena Tutubalina ${ }^{1}$ and Sergey Nikolenko ${ }^{1,2}$ \\ ${ }^{1}$ Kazan (Volga Region) Federal University, Kazan, Russia \\ ${ }^{2}$ Steklov Institute of Mathematics, St. Petersburg, Russia \\ Correspondence should be addressed to Elena Tutubalina; tutubalinaev@gmail.com
}

Received 14 April 2017; Accepted 27 July 2017; Published 5 September 2017

Academic Editor: Arindam Bit

Copyright ( 2017 Elena Tutubalina and Sergey Nikolenko. This is an open access article distributed under the Creative Commons Attribution License, which permits unrestricted use, distribution, and reproduction in any medium, provided the original work is properly cited.

\begin{abstract}
Adverse drug reactions (ADRs) are an essential part of the analysis of drug use, measuring drug use benefits, and making policy decisions. Traditional channels for identifying ADRs are reliable but very slow and only produce a small amount of data. Text reviews, either on specialized web sites or in general-purpose social networks, may lead to a data source of unprecedented size, but identifying ADRs in free-form text is a challenging natural language processing problem. In this work, we propose a novel model for this problem, uniting recurrent neural architectures and conditional random fields. We evaluate our model with a comprehensive experimental study, showing improvements over state-of-the-art methods of ADR extraction.
\end{abstract}

\section{Introduction}

Recent studies on text mining applications increasingly employ nonstandard sources of information to obtain new data related to health conditions, the efficiency of treatment, drug reactions, and interactions between different drugs. Users provide information about themselves through social media posts and free-text forum comments. This rich source of information has been successfully used, for instance, to monitor adverse drug reactions, making it possible to detect rare and underestimated reactions through the users complaining about their health [1].

In this work, we focus on the identification of adverse drug reactions (ADRs). ADRs are an essential part of drug postmarketing surveillance. Traditionally, reports about ADRs have been identified using (i) FDA's Adverse Event Reporting System (AERS) complaints from individual patients and their physicians and (ii) scientific literature and reports on clinical trials $[2,3]$. Nowadays, drug reactions can be extracted from user reviews provided on the Web, and processing this information in an automated way represents a novel and exciting approach to personalized medicine and wide-scale drug tests. Our goal is to extract phrases about ADRs in the context of a user's post. For example, a sentence "1st pill taken with food, a few hours after I experienced shortness of breath, a sense of depression, cramping, upset stomach" contains four ADRs, namely, shortness of breath, depression, cramping, and upset stomach. Formally, this challenging task is divided into the two subtasks: identification of ADRs and normalization of ADRs. In this paper, we focus on the first subtask.

Bidirectional recurrent neural networks (RNN) and conditional random fields (CRF) are considered to be among the most powerful models for sequence modeling [4-13], each one having its own advantages and disadvantages. In a direct RNN application, especially with LSTM or GRU cells, one can get a better model for long sequences of inputs, but the RNN output (a softmax layer) will classify every tag independently. CRF can solve this problem but is less expressive than RNN in modeling the sequence itself; therefore, it is natural to try to unite the two.

In this work, we apply a combination of RNN and CRF using the following strategy. We feed word-level representations into a bidirectional RNN to encode the context vectors 
for each word. On top of this RNN, we use a sequential CRF to jointly decode the words' labels for the entire sentence. A similar strategy has been successfully proposed in the past for two sequence labeling tasks: part-of-speech (POS) tagging and named entity recognition (NER) [14-16]. We evaluate our model for ADR extraction on an annotated corpus CADEC. The CADEC corpus consists of 1250 medical forum posts taken from AskaPatient.com [17], where each post has been manually annotated with mentions of ADRs. Our results show that the joint model of RNN and CRF improves the performance of state-of-the-art CRF and RNN models trained separately. Hence, we can summarize the contributions of this work as follows: (i) we have introduced a joint model that combines CRF and RNN to model the sequence of labels for ADR extraction; (ii) we have conducted empirical evaluation of this model on benchmark datasets; and (iii) experimental results have shown that the proposed model improves over state-of-the-art performance.

The paper is organized as follows: in Section 2, we survey related work; Section 3 introduces the combined RNN + CRF model, and Section 4 considers in detail our experimental evaluation. We conclude in Section 5.

\section{Related Work}

Our work represents a new look at the recently popular studies on biomedical text mining and pharmacovigilance from social media.

2.1. Biomedical Text Mining. Recent studies in various fields of biomedical research have applied text mining, including such problems as named entity recognition $[10,11]$, relation extraction $[18,19]$, text classification $[9,20]$, hypothesis generation $[21,22]$, and the creation of knowledge sources and linguistic resources. A comprehensive review of important areas of biomedical text mining can be found in [23, 24]. Huang and $\mathrm{Lu} \mathrm{[23]} \mathrm{reported} \mathrm{a} \mathrm{series} \mathrm{of} \mathrm{evaluations} \mathrm{of} \mathrm{natural}$ language processing (NLP) systems for various biomedical tasks, including both knowledge-based methods and machine learning approaches to NLP.

In general, biomedical named entities include genes/proteins, chemicals, drugs, and diseases. As for relations, most research studies have focused on the entities' functions (e.g., gene functions), relational events, and interactions (e.g., drug-drug or protein-protein interactions). Many studies have employed simple classifiers to extract information from texts. For example, Ngo et al. [25] employed a classification method on a set of features based on distributed representations to predict drug-disease relations in cancer treatment. Rastegar-Mojarad et al. [26] used machine learning methods to identify disease names from user reviews for about top 180 most frequently searched medications on the WebMD forum. In order to identify candidates for drug repurposing, the authors removed indications of known drugs and did a manual review of the comments without using FDA reports. The main limitation of this work is the lack of an annotated corpus to evaluate the proposed system. Zhang et al. [20] proposed a weighted average ensemble of four classifiers, based respectively on a handmade lexicon, $n$-grams, and word representation vectors (also called word embeddings). Avillach et al. [3] developed a method to find previously established relationships between drugs and adverse events using the MEDLINE corpus and medical subject headings and subheadings such as "chemically induced" and "adverse effects." Well-recognized limitations of these resources include the need of volunteers to report events and lack of sufficiently large result sets to draw the statistical conclusion. These drawbacks have led to the rise of pharmacovigilance from social media.

2.2. Pharmacovigilance from Social Media. Social media has been increasingly used for medical and pharmacological research since the early 2010s; the term "pharmacovigilance" was coined for automated monitoring of social media for potentially adverse drug effects and interactions.

NLP techniques have been applied in five main domain of texts: (i) biomedical literature, clinical trial records, and electronic medical/health records (e.g., medical correspondence and letters) $[3,5,10,27-30]$; (ii) short messages from Twitter [9, 31, 32]; (iii) user reviews from health-related and e-commerce websites [4, 26, 33, 34]; (iv) web search logs [22]; and (v) forum discussions and message boards about medications, health conditions, treatment modality, and so on [35-37]. Most of these works focused on creating linguistic methods based on keywords for extracting major adverse effects, classifiers to detect whether a text contains ADRs or is relevant to drug reactions, and sequence labeling algorithms to extract mentions of ADRs. A review of techniques applied to drug reaction detection has been given in $[38,39]$.

In opinion mining, one of the major tasks is the identification of opinion targets (also called aspects) or opinion expressions. This task has been studied by many researchers using frequency-based methods and unsupervised and supervised methods. In [40], authors described linguistic resources for event extraction: linguistics databases and vocabularies such as thesauri. Currently, most of the state-of-the-art methods are based on CRF with a set of hand-crafted features and bidirectional RNNs [7, 8, 10]. Irsoy and Cardie [7] applied deep RNNs to extract direct or expressive subjective expressions; in their experiments, 3-layer RNN outperformed CRF, semi-CRF, and 1-layer (i.e., shallow) RNN. Liu et al. [8] applied RNNs for aspect extraction from data sets about laptops and restaurants, and RNNs based on pretrained word embeddings outperformed feature-rich CRF-based models.

In recent years, there has been a growing interest in the area of detecting ADRs from social media. It started in 2010 with a pioneering study of Leaman et al. [41], who analyzed user posts regarding six drugs from the health-related social network DailyStrength. FDA alerts were used as a gold standard to evaluate discovered associations between drugs and ADRs. Yang et al. [42] conducted an experiment for ten drugs and five ADRs to examine associations between them on texts from online healthcare communities using association mining techniques. Rastegar-Mojarad et al. [26] developed a rule-based system to extract drug effects. Feldman et al. [37] identified ADRs on texts from healthrelated online forums. They employed dictionary-based drug 
detection, and symptoms were extracted with a combination of dictionary-based and pattern-based methods. Pointwise mutual information (PMI) was computed to evaluate the likelihood of a drug-ADR relation. The authors analyzed several case studies of drugs to show that some ADRs were reported prior to the FDA communication. One limitation of this work is the amount of annotated data; the test set contained less than 500 samples. See [39] for a comprehensive review of ADR extraction from social media data with NLP-based approaches.

Supervised machine learning techniques have been successfully applied to detect ADRs. Bian et al. [31] utilized an SVM classifier to identify tweets describing ADRs. YomTov and Gabrilovich [22] analyzed web search query logs to extract information related to drugs and adverse reactions. ADR extraction has been regarded in many studies as a sequence labeling problem using conditional random fields (CRF). CRFs with a rich set of contextual, lexicon-based, grammatical, and semantic features were used in $[6,9,33]$. In [6], the semantic features were based on word clusters using $k$-means clustering on pretrained word embeddings. A set of experiments showed that contextual and semantic features are the most effective to classify ADRs in tweets. We also note a Social Media Mining Shared Task Workshop (organized as part of the Pacific Symposium on Biocomputing 2016) devoted to mining pharmacological and medical information from Twitter, with a competition based on a published dataset [32].

Supervised models tend to work well when trained on fully labeled data. Although there is a large amount of unlabeled data from social media, labeled data are timeconsuming to obtain. Gupta et al. [35, 43] used semisupervised learning of patterns to identify drugs, symptoms, and conditions. Lexico-syntactic patterns have been learned with a seed dictionary of terms, and a bootstrapped rule-based method extracted specific entities that were missing from the seed dictionaries. One limitation of this approach is that it does not identify long descriptive phrases. Stanovsky et al. [44] employed an active learning technique to create a bootstrap lexicon of ADRs. The main advantage of this approach is that it can identify entities with a small number of hand-written rules or hand-labeled examples. We mark these works as possibilities for future improvements of this area.

The most relevant studies to the present paper are the works by Metke-Jimenez and Karimi [33], Miftahutdinov et al. [4], and Stanovsky et al. [44]; all of them used the CADEC corpus for training and testing. Metke-Jimenez and Karimi [33] applied dictionary-based methods and CRFs to identify ADRs from the CADEC corpus. They used baseline features, including a bag of words, letter $n$-grams, and word shapes (e.g., if the token composed of uppercase letters). For evaluation, they applied strict and relaxed versions of the evaluation for each matching span. The authors divided the corpus into training and testing sets, using a 70/ 30 split. CRF outperformed knowledge-based methods on the sentence level and achieved strict and relaxed $F_{1}$ measures of $60.2 \%$ and $84.9 \%$, respectively. Miftahutdinov et al. [4] applied CRF with a rich set of features to extract all disease-related entities including drug indications, ADRs, and patient history. For CRF features, they used hand-crafted features including contextual features, dictionaries, and cluster-based and distributed word representations. CRF outperformed bidirectional 2-layer and 3-layer RNNs on review level based on 5-fold cross evaluation and achieved $F_{1}$-measures of $69.1 \%$ and $79.4 \%$ on recognition of disease-related expressions in the exact and partial matching exercises, respectively. They used word embeddings trained on social media and on scientific literature separately. Stanovsky et al. [44] employed RNN and word embeddings trained on a Blekko medical corpus in conjunction with embeddings trained on DBpedia. If a word was a lexical match with one of the DBpedia entities, then the DBpedia embedding was used as the input of RNN. Otherwise, Blekko embedding was used. The authors used a 75/25 split and computed evaluation metrics for every word in a sentence instead of extracted spans of ADRs. The knowledge-infused RNN achieved $F_{1}$-measures of $93 \%$ in the evaluation of each word. The authors did not evaluate RNN in comparison with CRF and LSTM in comparison with GRU. We also note that those papers did not analyze the number of epochs for training RNNs and did not apply the joint model of RNN and CRF.

Our work differs from the mentioned works in several important aspects.

(1) We experiment with a joint model of CRF and RNN as well as both models separately.

(2) In addition, we employ CNN to extract characterlevel features instead of engineering of hand-crafted features.

(3) We use word embeddings trained on social media and on scientific literature.

(4) We present quantitative analysis as well as qualitative analysis of extracted ADRs to demonstrate variation in ADRs across different patient groups.

\section{Model}

This section illustrates our supervised model combining recurrent neural network (RNN) and conditional random fields (CRF) to the extraction of ADRs. We formulate the disease-related entity extraction as a sequence labeling problem. In the following subsections, we describe the architecture of the model.

3.1. Recurrent Neural Networks. RNNs are naturally used for sequence learning, where both input and output are word and label sequences. RNN has recurrent hidden states, which aim to simulate memory, that is, the activation of a hidden state at every time step depends on the previous hidden state [45]. The recurrent unit computes a weighted sum of the input signal.

Training RNNs to capture long-term dependencies is difficult due to the effect of vanishing gradients [46], so the most widely used modification of RNN units is the long short-term memory (LSTM) [47] that provides the "constant error 


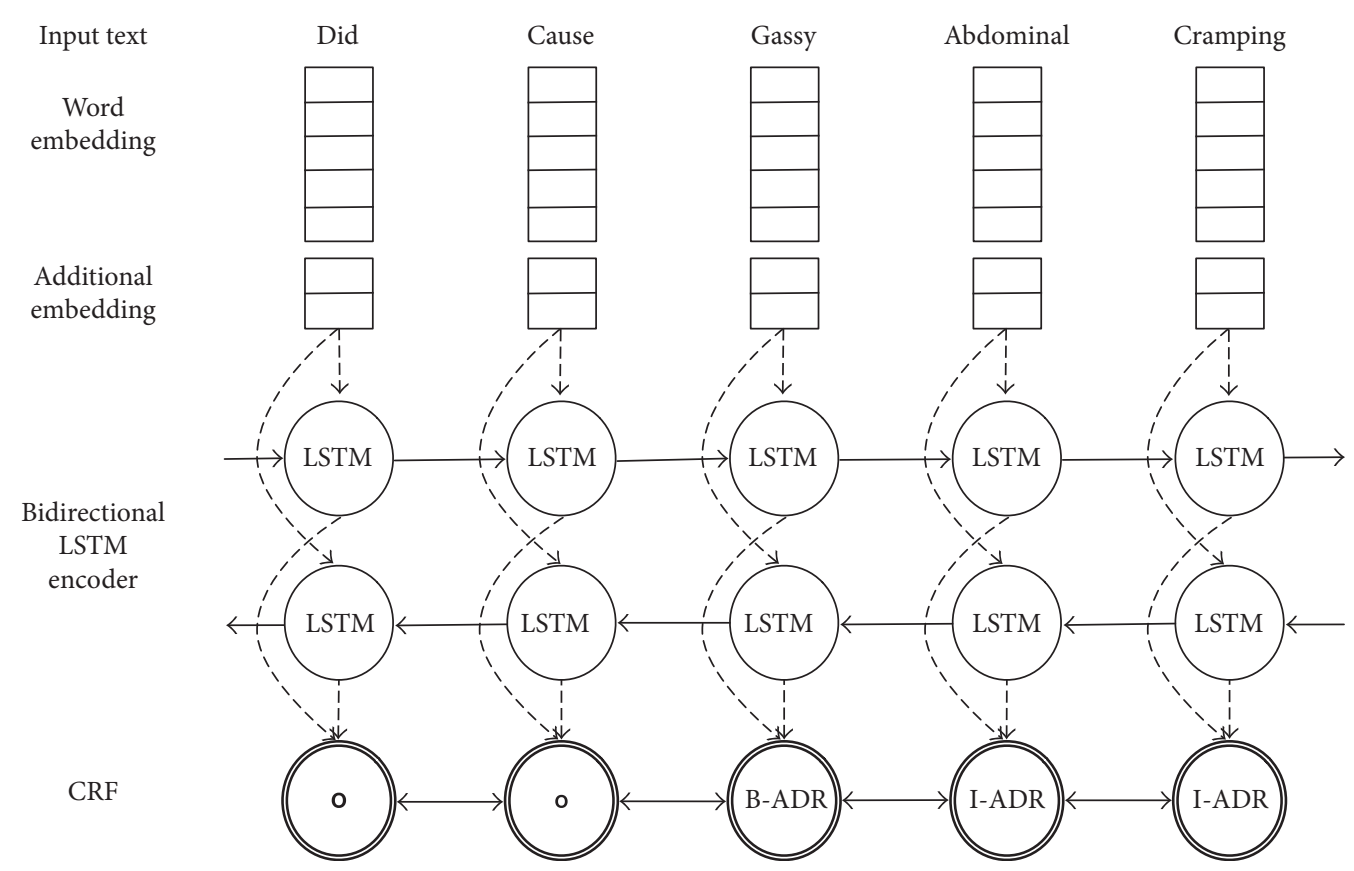

FIGURE 1: The main architecture of our model. Word embeddings are given as input to the bidirectional LSTM network. Dashed arrows represent the input and output vectors of the network with dropout. The labels follow the BIO (Beginning Inside Outside) tagging scheme.

carousel" and does not preclude free gradient flow. The most common LSTM architecture contains three gates: input gate, forget gate, and output gate, together with a recurrent cell. LSTM cells are usually organized in a chain, with outputs of previous LSTMs connected to the inputs of subsequent LSTMs. A recent simplification of the LSTM architecture is given by gated recurrent units (GRU) introduced by Cho et al. [48]. GRU is very similar to an LSTM cell; GRU has a single "update gate" instead of separate forget and input gates, does not distinguish cell state and hidden state, and always exposes the entire hidden state, without a special gate for it.

An important modification of the basic RNN architecture is bidirectional RNNs, where the past and the future context is available on every time step [49]. Bidirectional LSTMs, developed by Graves and Schmidhuber [50, 51], contain two chains of LSTM cells flowing in both forward and backward direction, and the final representation is either a linear combination or simply concatenation of their states.

3.2. Conditional Random Fields. CRF [52] is one of the stateof-the-art methods that takes a sequence of tokens as input, estimates the probabilities of labels (from a predefined set), and returns the best scoring label sequence. We denote by $x_{1}, \ldots, x_{n}, x_{i} \in R^{m}$ corresponding to the input sequence and by $Y$ to the labels. The CRF is defined by a graph whose vertices are indexes of $Y$ and edge weights correspond to the effects that $X$ and $Y$ have on each other, given that the Markov property holds. A linear-chain CRF is a CRF with a simple chain as the graph, where each edge has the form of $(j-1, j)$.

As shown in [52], the conditional probability of a label sequence is computed as follows:

$$
p_{\lambda, \mu}(y \mid x)=\frac{1}{Z(x)} \cdot \prod_{t=1}^{n} \exp \left(\lambda_{y_{t-1} y_{t}}+\left\langle\mu_{y_{t}}, x_{t}\right\rangle\right)
$$

where $Z(x)$ is the normalization, $x$ is the feature vector, $\mu$ is the matrix of size $|Y| \times m, \lambda$ is the matrix of $|Y| \times|Y|$, and $\mu_{y_{t}}$ is the $y_{t}$ row in the matrix $\mu$. In the equation, the augend represents the score of a transition from the tag $y_{t-1}$ to the tag $y_{t}$. In our case, the addend represents the score of the tag $y_{t}$ of the $t$ th word. We define the addend to be the matrix of scores output by the recurrent network. Maximum likelihood learning involves maximizing

$$
y=\arg \max _{y \in Y} \frac{1}{Z(x)} \cdot \prod_{t=1}^{n} \exp \left(\lambda_{y_{t} y_{t-1}}+\left\langle\mu_{y_{t}}, x_{t}\right\rangle\right) .
$$

We use an implementation of the linear-chain CRF that minimizes the loss function and trains the weights for computing the global tag sequence scores. During testing, the model applies the Viterbi algorithm to predict the best scoring tag sequence.

3.3. Joint Model. The main idea of our proposed model is to combine CRF with a neural network, using nonlinear potentials modeled by a neural network instead of linear potential functions based on sparse features. Figure 1 illustrates the proposed architecture for ADR extraction.

First, word embeddings are fed into the bidirectional RNN (e.g., LSTM). Circles represent LSTM cells. The network returns a representation of the forward and backward context for each word. Then, these output vectors go through a dropout layer for regularization [53]. The result feeds into a dense layer with linear activation, whose output size equals the number of tags. The difference with standard RNN 
architecture is that we do not use the softmax output from this layer directly but rather utilize the output of the dense layer for an additional CRF layer to jointly decode the sequence of context tags.

Another important part of the model is the extra vector marked as "additional embedding" on Figure 1. In the experiments shown below, we augmented the basic word embeddings with an additional vector trained with a character-level CNN [16], simply concatenating the two vectors as input for the bidirectional LSTM; we will see that this additional model also improves the final results.

\section{Experiments and Discussion}

4.1. Quality Metrics and Datasets. In this section, we evaluate our model and compare it with baseline approaches. Since the boundaries of expressions are hard to define even for human annotators [54], we follow [55, 56] and conduct the experimental evaluation as follows:

(1) Exact matching following CoNLL evaluation [57]

(2) Partial matching as described in [56].

We computed several model accuracy metrics such as macroaveraged precision $(P)$, recall $(R)$, and $F_{1}$-measure $(F)$ as follows:

$$
\begin{aligned}
& P=\frac{\mathrm{TP}}{\mathrm{TP}+\mathrm{FP}}, \\
& R=\frac{\mathrm{TP}}{\mathrm{TP}+\mathrm{FN}}, \\
& F=\frac{2 \cdot P \cdot R}{P+R},
\end{aligned}
$$

where TP is the number of correctly predicted annotations and FP and FN are the numbers of false positives and false negatives, respectively. Following [56], we used the following formulas for partial matching:

$$
\begin{aligned}
& P=\frac{\left|t \cap t_{s}\right|}{\left|t_{s}\right|}, \\
& R=\frac{\left|t \cap t_{s}\right|}{|t|},
\end{aligned}
$$

where $t_{s}$ is an extracted term which intersects with the term $t$, $t_{s} \cap t$ is the intersection between $t$ and $t_{s}$, and $|t|$ is the length of this term in tokens. For partial matching, we calculated metrics for every sentence and averaged the resulting values.

We used the Keras library (https://keras.io/) to implement neural networks and the BIO (Beginning Inside Outside) tagging scheme on the sentence level. The batch size was 128; we used the Adam optimizer with default parameters [58]. We evaluated our network using the high-quality annotations from the CADEC corpus. Similar to [33], we excluded overlaps between spans of ADRs in the CADEC corpus, selecting the longest continuous span and combining these ADRs into a single annotation.

The corpus was split into two different datasets, leaving $70 \%$ for training (with a total of 875 reviews, 5264 sentences, and 3933 ADRs) and 30\% (375 reviews, 2356 sentences, and 1837 ADRs) for testing.

4.2. Experimental Results. We evaluate our model by comparing with the following methods:

(1) CRF with the following baseline features: each word itself with a part-of-speech tag, the suffixes and prefixes to 6 characters in length, and a window of two words in both directions (backward and forward) from the current word

(2) Feature-rich CRF-based approach with parameters as proposed in [4]; this method utilizes the following features: baseline contextual features, dictionaries, and cluster-based and distributed word representation. The authors used the following dictionaries: the Unified Medical Language System (UMLS), ADR lexicons, and a dictionary of multiword expressions such as "feel tired," and "feel sleepy." The Brown hierarchical algorithm was used for clusterbased word representations (vector size of 150). The authors trained Continuous Bag of Words model on a corpus of health-related reviews with the following parameters: vector size of 200, the length of local context of 10, negative sampling of 5, and vocabulary cutoff of 10 . We used publicly available implementation of this feature-rich approach (https://github. com/dartrevan/ChemTextMining/)

(3) Deep bidirectional RNNs with a softmax layer, in particular, LSTM and GRU, where the combination of the network's outputs is fed into a fully connected layer with softmax activation.

We used a maximum of 100 epochs to train each network. For fair comparison, all networks used the same word embeddings trained on 2.5 million of health-related reviews [4]. We found $97 \%$ of words in the vocabulary, and for $3 \%$ of words, the representations were uniformly sampled from the range of embedding weights [59]. The results of different methods are shown in Table 1.

Table 1 shows that the proposed model consistently outperforms other approaches in terms of both precision and $F$-measure, while staying roughly on par with the best recurrent models in terms of recall. Therefore, we conclude that a combination of RNN and CRF indeed leads to quality improvements for ADR extraction from free-text reviews. The second conclusion is that concatenating input word embeddings with an extra embedding vector based on a character-level CNN also significantly improves the results. Another interesting conclusion from Table 1 is that GRUbased recurrent architectures consistently outperform LSTM-based architectures in the exact matching exercise. Finally, another interesting conclusion is that $F_{1}$-scores of 3-layer $\mathrm{GRU}+\mathrm{CNN}+\mathrm{CRF}$ increased from $70.65 \%$ to $79.78 \%$ in the partial matching as compared to the exact exercise due to boundary problems. Qualitative analysis of results indicates errors associated with boundaries of entities due to the presence of negations (e.g., "I have no pain"), 
TABLE 1: Results of the proposed models and baseline methods.

\begin{tabular}{|c|c|c|c|c|c|c|}
\hline \multirow{2}{*}{ Method } & \multicolumn{3}{|c|}{ Exact } & \multicolumn{3}{|c|}{ Partial } \\
\hline & $P$ & $R$ & $F$ & $P$ & $R$ & $F$ \\
\hline Baseline CRF & 0.6254 & 0.5972 & 0.6110 & 0.8145 & 0.7539 & 0.7521 \\
\hline Feature-rich CRF & 0.6726 & 0.6532 & 0.6628 & 0.8303 & 0.7646 & 0.7622 \\
\hline 1-layer LSTM & 0.5798 & 0.6587 & 0.6167 & 0.8121 & 0.8065 & 0.7809 \\
\hline 2-layer LSTM & 0.6362 & 0.7044 & 0.6686 & 0.8090 & 0.8495 & 0.8005 \\
\hline 3-layer LSTM & 0.6588 & 0.7022 & 0.6798 & 0.8247 & 0.8323 & 0.7997 \\
\hline 4-layer LSTM & 0.6689 & 0.7093 & 0.6885 & 0.8255 & 0.8280 & 0.8000 \\
\hline 1-layer GRU & 0.5862 & 0.6772 & 0.6284 & 0.7995 & 0.8368 & 0.7900 \\
\hline 2-layer GRU & 0.6384 & 0.7093 & 0.6720 & 0.8165 & 0.8338 & 0.8002 \\
\hline 3-layer GRU & 0.6675 & 0.7191 & 0.6923 & 0.8151 & 0.8373 & 0.8009 \\
\hline 4-layer GRU & 0.6565 & 0.7262 & 0.6896 & 0.8006 & 0.8665 & 0.8033 \\
\hline 2-layer LSTM + CRF & 0.6947 & 0.6973 & 0.6960 & 0.8191 & 0.8161 & 0.7872 \\
\hline 2-layer LSTM + CNN + CRF & 0.6809 & 0.7039 & 0.6922 & 0.8083 & 0.8488 & 0.7978 \\
\hline 3-layer LSTM + CNN + CRF & 0.6868 & 0.7066 & 0.6965 & 0.8270 & 0.8488 & 0.8115 \\
\hline 3-layer GRU + CNN + CRF & 0.7048 & 0.7082 & 0.7065 & 0.8219 & 0.8311 & 0.7978 \\
\hline
\end{tabular}

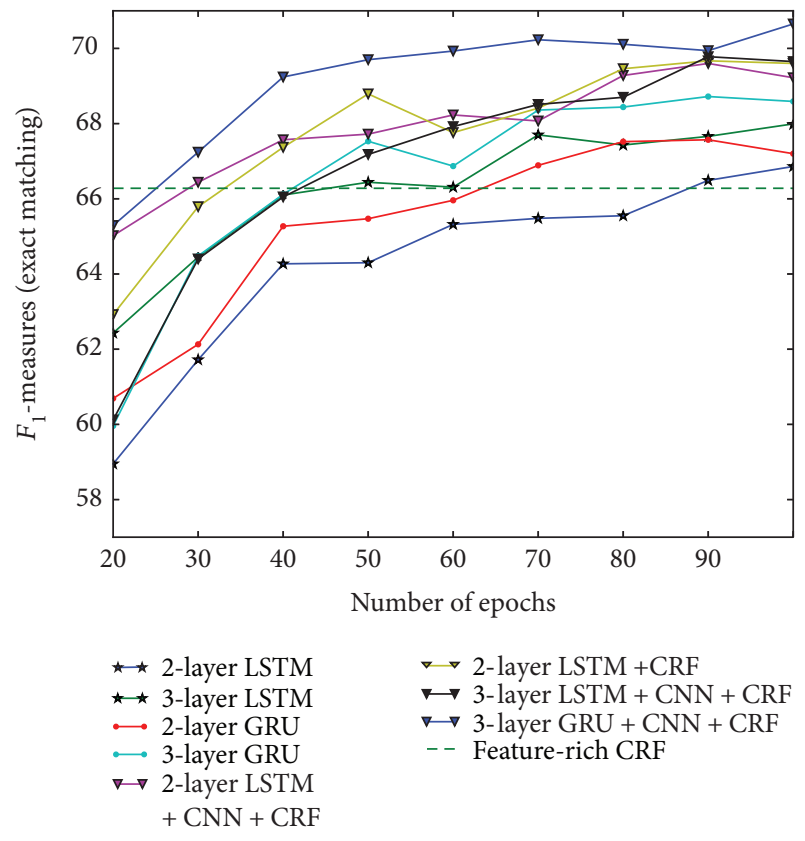

Figure 2: Performance on the testing data set or different number of training epochs.

conjunctions, verbs, adjectives, or adverbs (e.g., "lowered total cholesterol dramatically").

We initially set the number of epochs for training models to be 100 and explored the quality metrics for the number of training epochs ranging from 20 to 100 . Figure 2 presents the results. It shows that training of deep LSTM and GRU can be effectively achieved at around 60-80 epochs before the performance becomes stable. The joint model of 2-layer $\mathrm{LSTM}+\mathrm{CNN}+\mathrm{CRF}$ and 3-layer GRU + CNN + CRF outperformed CRF starting at 30-40 epochs, and from here on, performance improved slowly.
4.3. Qualitative Analysis of Extracted ADR Mentions. Adverse drug reactions can differ significantly depending on the patient. To investigate the difference between adverse effects for various drugs, we collected reviews from a health information service webmd.com. Each review contains the following fields:

(1) Brand name of a drug used to treat this disease

(2) Condition/reason for taking treatment

(3) The free-text review given for the effects caused by the use of the drug

(4) Demographic information about the author of this review (age and gender).

We also note that such demographic information is not commonly provided in discussion groups and websites. In a recent study [60], several approaches to automated mining of demographic information from texts about drugs were evaluated including neural networks, supervised machine learning, and topic modeling.

We selected reviews about the following health conditions for analysis:

(1) 4,563 reviews about depressive disorder (drugs: Cymbalta, Lexapro, Xanax, Zoloft, or Prozac)

(2) 5,422 reviews about high blood pressure (drugs: lisinopril, atenolol, Bystolic, Diovan, and hydrochlorothiazide)

(3) 10,914 reviews about fibromyalgia (drugs: Cymbalta, Lyrica, tramadol, Prozac, amitriptyline, Savella, Paxil CR, Ultram, Paxil, cyclobenzaprine).

In order to detect ADRs related to a particular demographic group, we extracted all ADRs that appeared in 
TABLE 2: ADRs extracted from reviews for the drugs that treat depression.

\begin{tabular}{|c|c|}
\hline Group & Adverse drug reactions \\
\hline All authors & $\begin{array}{l}\text { Anxiety, depression, panic attacks, depressed, pain, weight gain, nausea, headaches, dizziness, insomnia, } \\
\text { dizzy, mood swings, tired, dry mouth, sweating }\end{array}$ \\
\hline Gender group "female" & $\begin{array}{l}\text { Rash, gained weight, could not sleep, heartburn, severe nausea, lost weight, restless, very irritable, heart racing, } \\
\text { disconnected, stiffness, upset, severe migraines, cramping, neck pain, twitching, fever, skin problems }\end{array}$ \\
\hline Gender group "male" & $\begin{array}{l}\text { Erectile dysfunction, pins and needles, burning sensations, loose bowels, urination, uneasiness, trouble with } \\
\text { dizziness, severe drowsiness, night sweat, chest pressure, blisters, clammy hands }\end{array}$ \\
\hline Age group "19-34" & $\begin{array}{l}\text { Couldn't sleep, anger issues, loss of sex drive, cramps, unmotivated, jaw pain, frequent headaches, fever, } \\
\text { stomach pains, crying for no reason, severe dizziness, intrusive thoughts }\end{array}$ \\
\hline Age group “45-64” & $\begin{array}{l}\text { Nervous breakdown, aches and pains, swelling, muscle aches, delayed ejaculation, profuse sweating, } \\
\text { indigestion, ringing in my ears, spasms, trouble urinating, palpitations }\end{array}$ \\
\hline
\end{tabular}

TABLE 3: ADRs extracted from reviews for the drugs that treat high blood pressure.

\begin{tabular}{ll}
\hline Group & Adverse drug reactions \\
\hline All authors & $\begin{array}{l}\text { Cough, coughing, dizziness, dizzy, headaches, dry cough, fatigue, tired, headache, weight gain, hair loss, nausea, } \\
\text { anxiety, shortness of breath, tiredness, diarrhea, chest pain, depression, joint pain, rash, swelling, very tired, light } \\
\text { headed, blurred vision }\end{array}$ \\
$\begin{array}{l}\text { Gender group } \\
\text { "female" }\end{array}$ & $\begin{array}{l}\text { Heart palpitations, hives, gagging, hot flashes, extremely tired, nightmares, chronic cough, cold hands and feet, } \\
\text { panic attacks, exhausted, weight loss, blurry vision, heartburn, sleepy, persistent cough, severe headaches, stomach } \\
\text { pain, numbness } \\
\text { Age group "45-64" }\end{array}$ \\
Bloating, muscle aches, persistent cough, indigestion, stomach pain, post nasal drip, sick, lack of sleep, ringing in my \\
ears, stomach pains, foot cramps, tightness in chest, falling out, severe coughing, faint, nagging cough, no energy \\
Age group "25-44"
\end{tabular}

TABLE 4: ADRs extracted from reviews for the drugs that treat fibromyalgia.

\begin{tabular}{|c|c|}
\hline Group & Adverse drug reactions \\
\hline All authors & $\begin{array}{l}\text { Pain, depression, anxiety, weight gain, nausea, headaches, depressed, dizziness, dizzy, panic attacks, nerve pain, } \\
\text { insomnia, dry mouth, constipation, sweating, tired, headache, fatigue, back pain, mood swings, hot flashes, nightmares, } \\
\text { suicidal thoughts, severe pain, blurred vision, muscle pain, vomiting, chronic pain, suicidal, neuropathic pain, } \\
\text { drowsiness, trouble sleeping, sex drive, diarrhea, seizures, crying, anxious, nauseous, numbness, swelling, leg pain, } \\
\text { night sweats, vertigo, tremors, joint pain, itching, burning, panic attack, sleepiness, drowsy }\end{array}$ \\
\hline $\begin{array}{l}\text { Gender group } \\
\text { "female" }\end{array}$ & $\begin{array}{l}\text { Severe migraines, water retention, severe panic attacks, suicidal ideation, exhaustion, stiff, inability to sleep, rapid } \\
\text { heartbeat, crazy dreams, sweaty, nervous breakdown, extreme sweating, fogginess, flushing, major weight gain, } \\
\text { increased my appetite }\end{array}$ \\
\hline $\begin{array}{l}\text { Gender group } \\
\text { "male" }\end{array}$ & $\begin{array}{l}\text { Blisters, premature ejaculation, foot neuropathy, burning discomfort, can barely walk, pain in my toes, anger problems, } \\
\text { loss of libido, pancreatitis, pain in lower back, hiccups, shock sensations, couldn't walk, can't walk, panic problems, } \\
\text { "shock" sensations, hangover, short-term memory, severe trouble urinating }\end{array}$ \\
\hline
\end{tabular}

reviews more than four times and then excluded ADRs if the exact match appears in reviews of authors with a different demographic tag (e.g., "male"/"female" or "age 19-34" over other ages). Tables 2, 3, and 4 present the results. The tables indicate that key adverse reactions change with age or gender, reflecting quite natural progressions that match well with medical and commonsense intuition. Hence, our method can also be used to mine qualitative information from a dataset of medical reviews, perhaps uncovering new ADRs in a certain user group.

\section{Conclusion}

In this work, we have proposed a novel approach to extracting adverse drug reactions from user reviews: a combination of a bidirectional LSTM-based recurrent neural network and a CRF that operates on the scores extracted by this neural network. We have evaluated our approach against state-ofthe art neural models on a representative ADR extraction dataset and have found that the results have improved significantly. Moreover, further improvements were obtained by 
extending input embeddings with a character-level model. Thus, our final model successfully combines three different approaches to statistical modeling in NLP. In further work, we plan to experiment with other neural models in similar general architectures and further improve the state of the art in ADR extraction from free-text reviews.

\section{Conflicts of Interest}

The authors declare that they have no conflicts of interest.

\section{Acknowledgments}

The work was supported by the Russian Science Foundation Grant no. 15-11-10019.

\section{References}

[1] I. Segura-Bedmar, P. Martínez, R. Revert, and J. MorenoSchneider, "Exploring Spanish health social media for detecting drug effects," BMC Medical, vol. 15, Supplement 2, p. S6, 2015.

[2] M. S. Boguski, K. D. Mandl, and V. P. Sukhatme, "Repurposing with a difference," Science, vol. 324, no. 5933, pp. 1394-1395, 2009.

[3] P. Avillach, J.-C. Dufour, G. Diallo et al., "Design and validation of an automated method to detect known adverse drug reactions in MEDLINE: a contribution from the EU-ADR project," Journal of the American Medical Informatics Association, vol. 20, no. 3, pp. 446-452, 2013.

[4] Z. S. Miftahutdinov, E. V. Tutubalina, and A. E. Tropsha, Identifying Disease-Related Expressions in Reviews Using Conditional Random Fields, Komp'juternaja Lingvistika i Intellektual'nye Tehnologii, 2017.

[5] A. N. Jagannatha and H. Yu, "Bidirectional RNN for medical event detection in electronic health records," Proceedings of the Conference Association for Computational Linguistics, vol. 2016, pp. 473-482, 2016.

[6] A. Nikfarjam, A. Sarker, K. O'Connor, R. Ginn, and G. Gonzalez, "Pharmacovigilance from social media: mining adverse drug reaction mentions using sequence labeling with word embedding cluster features," Journal of the American Medical Informatics Association, vol. 22, no. 3, pp. 671-681, 2015.

[7] O. Irsoy and C. Cardie, "Opinion mining with deep recurrent neural networks," in Proceedings of the 2014 Conference on Empirical Methods in Natural Language Processing (EMNLP 2014): A meeting of SIGDAT, a Special Interest Group of the ACL, pp. 720-728, Doha, Qatar, October 2014.

[8] P. Liu, S. R. Joty, and H. M. Meng, "Fine-grained Opinion Mining with Recurrent Neural Networks and Word Embeddings," in Proceedings of the 2015 Conference on Empirical Methods in Natural Language Processing (EMNLP 2015), pp. 1433-1443, Lisbon, Portugal, September 2015.

[9] W. Wang, "Mining adverse drug reaction mentions in twitter with word embeddings," in Proceedings of the Social Media Mining Shared Task Workshop at the Pacific Symposium on Biocomputing, 2016.

[10] H.-C. Lee, Y.-Y. Hsu, and H.-Y. Kao, "An enhanced CRFbased system for disease name entity recognition and normalization on BioCreative V DNER task," in Proceedings of the Fifth BioCreative Challenge Evaluation Workshop, pp. 226233, 2015, http://www.biocreative.org.
[11] Q. Wei, T. Chen, R. Xu, Y. He, and L. Gui, "Disease named entity recognition by combining conditional random fields and bidirectional recurrent neural networks," Database, vol. 2016, 2016.

[12] R. Gareev, M. Tkachenko, and V. Solovyev, Introducing Baselines for Russian Named Entity Recognition, 2013.

[13] V. A. Mozharova and N. V. Loukachevitch, "Combining knowledge and CRF-based approach to named entity recognition in Russian," in Analysis of Images, Social Networks and Texts, pp. 185-195, Springer, Cham, 2016.

[14] Z. Huang, W. Xu, and K. Yu, Bidirectional LSTM-CRF Models for Sequence Tagging, 2015, arXiv preprint arXiv:1508.01991.

[15] J. Chiu and E. Nichols, Named Entity Recognition with Bidirectional LSTM-CNNs, 2015, arXiv preprint arXiv:1511.08308.

[16] X. Ma and E. Hovy, End-to-End Sequence Labeling via BiDirectional LSTM-CNNs-CRF, 2016, arXiv [cs.LG].

[17] S. Karimi, A. Metke-Jimenez, M. Kemp, and C. Wang, "Cadec: a corpus of adverse drug event annotations," Journal of Biomedical Informatics, vol. 55, pp. 73-81, 2015.

[18] C.-H. Wei, Y. Peng, R. Leaman et al., "Overview of the BioCreative V chemical disease relation (CDR) task," Proceedings of the Fifth BioCreative Challenge Evaluation Workshop, pp. 154166, 2015, http://www.biocreative.org.

[19] J. Gu, L. Qian, and G. Zhou, "Chemical-induced disease relation extraction with various linguistic features," Database, vol. 2016, 2016.

[20] Z. Zhang, J.-Y. Nie, and X. Zhang, "An ensemble method for binary classification of adverse drug reactions from social media," Proceedings of the Social Media Mining Shared Task Workshop at the Pacific Symposium on Biocomputing.

[21] S. Spangler, A. D. Wilkins, B. J. Bachman et al., "Automated hypothesis generation based on mining scientific literature," Proceedings of the 20th ACM SIGKDD International Conference on Knowledge Discovery and Data Mining, pp. 18771886, ACM, New York, NY, USA, 2014.

[22] E. Yom-Tov and E. Gabrilovich, "Postmarket drug surveillance without trial costs: discovery of adverse drug reactions through large-scale analysis of web search queries," Journal of Medical Internet Research, vol. 15, no. 6, article e124, 2013.

[23] C.-C. Huang and Z. Lu, "Community challenges in biomedical text mining over 10 years: success, failure and the future," Briefings in Bioinformatics, vol. 17, no. 1, pp. 132-144, 2016.

[24] A. M. Cohen and W. R. Hersh, "A survey of current work in biomedical text mining," Briefings in Bioinformatics, vol. 6, no. 1, pp. 57-71, 2005.

[25] D. L. Ngo, N. Yamamoto, V. A. Tran et al., "Application of word embedding to drug repositioning," Journal of Biomedical Science and Engineering, vol. 9, p. 7, 2016.

[26] M. Rastegar-Mojarad, H. Liu, and P. Nambisan, "Using social media data to identify potential candidates for drug repurposing: a feasibility study," JMIR Research Protocols, vol. 5, no. 2, article e121, 2016.

[27] S. N. Deftereos, C. Andronis, E. J. Friedla, A. Persidis, and A. Persidis, "Drug repurposing and adverse event prediction using high-throughput literature analysis," Wiley Interdisciplinary Reviews: Systems Biology and Medicine, vol. 3, no. 3, pp. 323-334, 2011.

[28] T. C. Carter and M. M. He, "Challenges of identifying clinically actionable genetic variants for precision medicine," Journal of Healthcare Engineering, vol. 2016, Article ID 3617572, 14 pages, 2016. 
[29] A. Helmer, M. Lipprandt, T. Frenken, M. Eichelberg, and A. Hein, "3DLC: a comprehensive model for personal health records supporting new types of medical applications," Journal of Healthcare Engineering, vol. 2, no. 3, pp. 321-336, 2011.

[30] S. Saboor, A. Hoerbst, and E. Ammenwerth, "Quality of electronic health records-coverage of potential information weaknesses by major EHR quality seals," Journal of Healthcare Engineering, vol. 2, no. 3, pp. 365-388, 2011.

[31] J. Bian, U. Topaloglu, and F. Yu, "Towards large-scale twitter mining for drug-related adverse events," Proceedings of the 2012 International Workshop on Smart Health and Wellbeing, pp. 25-32, ACM, New York, NY, USA, 2012.

[32] A. Sarker, A. Nikfarjam, and G. Gonzalez, "Social media mining shared task workshop," Pacific Symposium Biocomputing, vol. 21, pp. 581-592, 2016.

[33] A. Metke-Jimenez and S. Karimi, Concept Extraction to Identify Adverse Drug Reactions in Medical Forums: A Comparison of Algorithms, 2015, arXiv [cs.AI].

[34] C. K. Reddy and C. C. Aggarwal, Healthcare Data Analytics, CRC Press, 2015.

[35] S. Gupta, D. L. MacLean, J. Heer, and C. D. Manning, "Induced lexico-syntactic patterns improve information extraction from online medical forums," Journal of the American Medical Informatics Association, vol. 21, pp. 902-909, 2014.

[36] A. Benton, L. Ungar, S. Hill et al., "Identifying potential adverse effects using the web: a new approach to medical hypothesis generation," Journal of Biomedical Informatics, vol. 44, pp. 989-996, 2011.

[37] R. Feldman, O. Netzer, A. Peretz, and B. Rosenfeld, "Utilizing text mining on online medical forums to predict label change due to adverse drug reactions," Proceedings of the 21th ACM SIGKDD International Conference on Knowledge Discovery and Data Mining, pp. 1779-1788, ACM, New York, NY, USA, 2015.

[38] S. Karimi, C. Wang, A. Metke-Jimenez, R. Gaire, and C. Paris, "Text and data mining techniques in adverse drug reaction detection," ACM Computing Surveys, vol. 47, pp. 56:1-56:39, 2015.

[39] A. Sarker, R. Ginn, A. Nikfarjam et al., "Utilizing social media data for pharmacovigilance: a review," Journal of Biomedical Informatics, vol. 54, pp. 202-212, 2015.

[40] V. Solovyev and V. Ivanov, "Knowledge-driven event extraction in Russian: corpus-based linguistic resources," Computational intelligence and neuroscience, vol. 2016, Article ID 4183760, 11 pages, 2016.

[41] R. Leaman, L. Wojtulewicz, R. Sullivan, A. Skariah, J. Yang, and G. Gonzalez, "Towards internet-age pharmacovigilance: extracting adverse drug reactions from user posts to health-related social networks," Proceedings of the 2010 Workshop on Biomedical Natural Language Processing, pp. 117-125, Association for Computational Linguistics, Stroudsburg, PA, USA, 2010.

[42] C. C. Yang, H. Yang, L. Jiang, and M. Zhang, "Social media mining for drug safety signal detection," Proceedings of the 2012 International Workshop on Smart Health and Wellbeing, pp. 33-40, ACM, New York, NY, USA, 2012.

[43] S. Gupta and C. D. Manning, Improved Pattern Learning for Bootstrapped Entity Extraction, 2014, CoNLL.

[44] G. Stanovsky, D. Gruhl, and P. N. Mendes, Recognizing Mentions of Adverse Drug Reaction in Social Media Using Knowledge-Infused Recurrent Models, 2017, EACL.

[45] J. L. Elman, "Finding structure in time," Cognitive Science, vol. 14, pp. 179-211, 1990.
[46] Y. Bengio, P. Simard, and P. Frasconi, "Learning long-term dependencies with gradient descent is difficult," IEEE Transactions on Neural Network, vol. 5, no. 2, pp. 157-166, 1994.

[47] K. Greff, R. K. Srivastava, J. Koutnik, B. R. Steunebrink, and J. Schmidhuber, "LSTM: a search space odyssey," IEEE Transactions on Neural Networks and Learning Systems, 2016.

[48] K. Cho, B. van Merrienboer, C. Gulcehre et al., "Learning phrase representations using RNN encoder-decoder for statistical machine translation," Proceedings of the 2014 Conference on Empirical Methods in Natural Language Processing (EMNLP), pp. 1724-1734, Association for Computational Linguistics, Stroudsburg, PA, USA, 2014.

[49] M. Schuster and K. K. Paliwal, "Bidirectional recurrent neural networks," IEEE Transactions on Signal Processing, vol. 45, pp. 2673-2681, 1997.

[50] A. Graves, S. Fernández, and J. Schmidhuber, "Bidirectional LSTM networks for improved phoneme classification and recognition," Artificial Neural Networks: Formal Models and Their Applications - ICANN 2005, pp. 799-804, Springer, Berlin, Heidelberg, 2005.

[51] A. Graves and J. Schmidhuber, "Framewise phoneme classification with bidirectional LSTM networks," Proceedings 2005 IEEE International Joint Conference on Neural Networks, vol. 4, pp. 2047-2052, 2005.

[52] J. Lafferty, A. McCallum, and F. Pereira, "Conditional random fields: probabilistic models for segmenting and labeling sequence data," Proceedings of the Eighteenth.

[53] N. Srivastava, G. E. Hinton, A. Krizhevsky, I. Sutskever, and R. Salakhutdinov, "Dropout: a simple way to prevent neural networks from overfitting," Journal of Machine Learning Research, vol. 15, pp. 1929-1958, 2014.

[54] J. Wiebe, T. Wilson, and C. Cardie, "Annotating expressions of opinions and emotions in language," Language Resources and Evaluation, vol. 39, pp. 165-210, 2006.

[55] O. Irsoy and C. Cardie, "Opinion mining with deep recurrent neural networks," Proceedings of the 2014 Conference on Empirical Methods in Natural Language Processing (EMNLP), pp. 720-728, Association for Computational Linguistics, Stroudsburg, PA, USA, 2014.

[56] N. Loukachevitch, P. Blinov, E. Kotelnikov, Y. Rubtsova, V. Ivanov, and E. Tutubalina, "SentiRuEval: testing objectoriented sentiment analysis systems in Russian," Proceedings of International Conference Dialog, pp. 3-13, 2015.

[57] E. F. Tjong Kim Sang and F. De Meulder, "Introduction to the CoNLL-2003 shared task: language-independent named entity recognition," Proceedings of the Seventh Conference on Natural Language Learning at HLT-NAACL 2003 - Volume 4, pp. 142147, Association for Computational Linguistics, Stroudsburg, PA, USA, 2003.

[58] D. Kinga and J. B. Adam, "A method for stochastic optimization," International Conference on Learning Representations (ICLR).

[59] K. He, X. Zhang, S. Ren, and J. Sun, "Delving deep into rectifiers: surpassing human-level performance on imagenet classification," in Proceedings of the IEEE International Conference on Computer Vision, pp. 1026-1034, Santiago, Chile, 2015.

[60] E. Tutubalina and S. Nikolenko, "Automated prediction of demographic information from medical user reviews," International Conference on Mining Intelligence and Knowledge Exploration, pp. 1-11, Springer, 2016. 


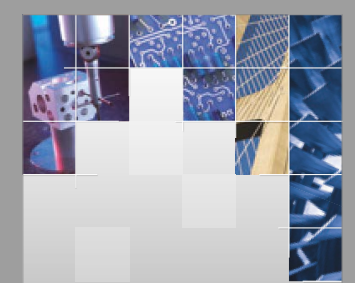

\section{Enfincering}
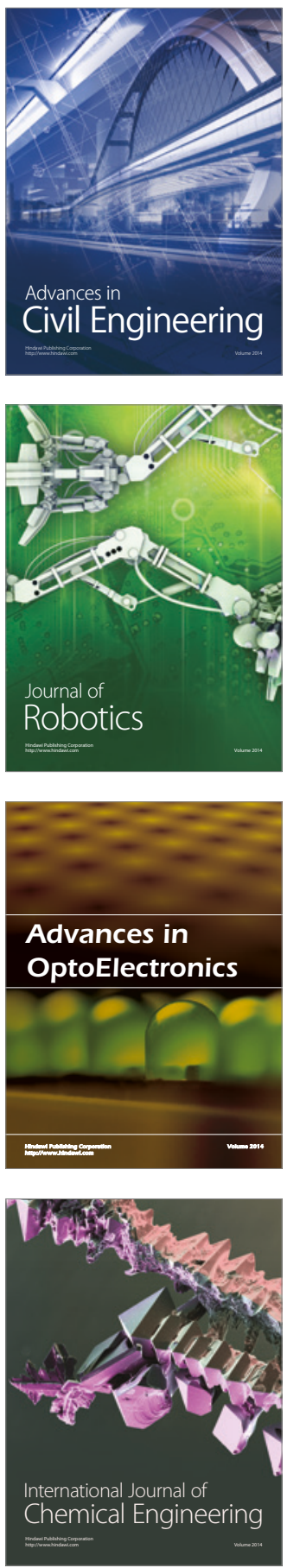

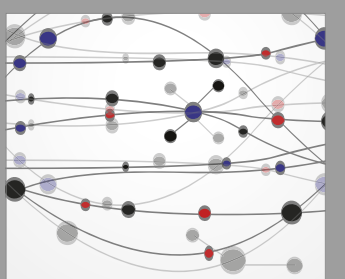

The Scientific World Journal

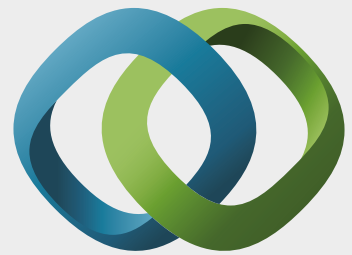

\section{Hindawi}

Submit your manuscripts at

https://www.hindawi.com
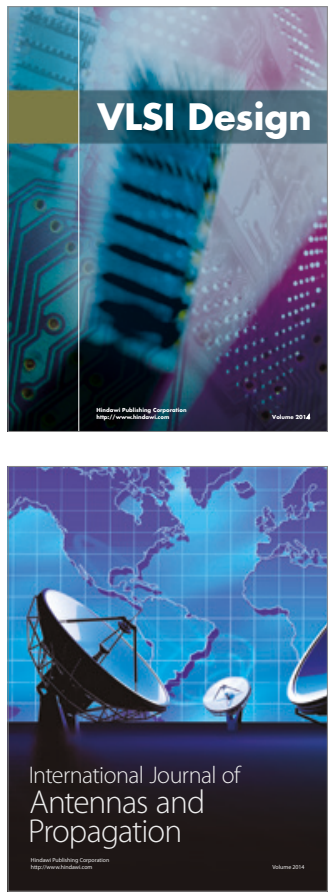

\section{Rotating}

Machinery
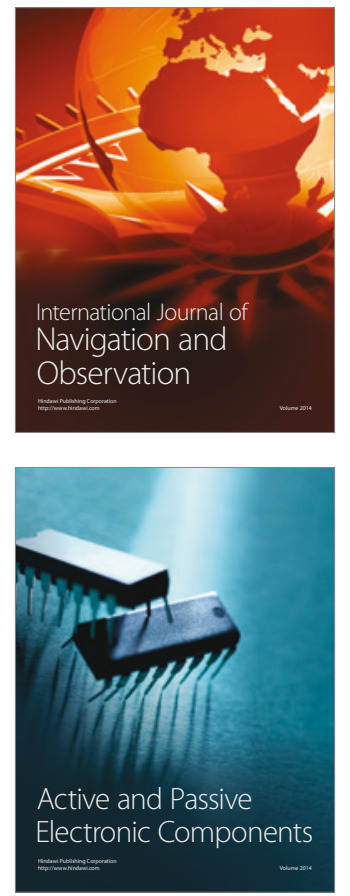
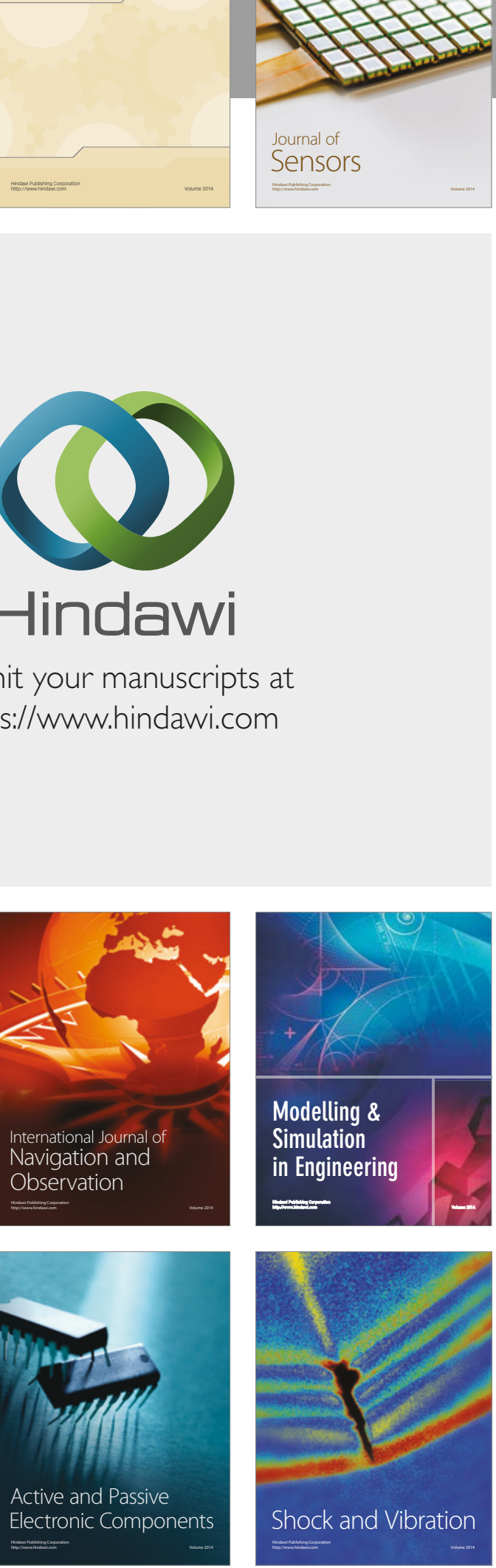
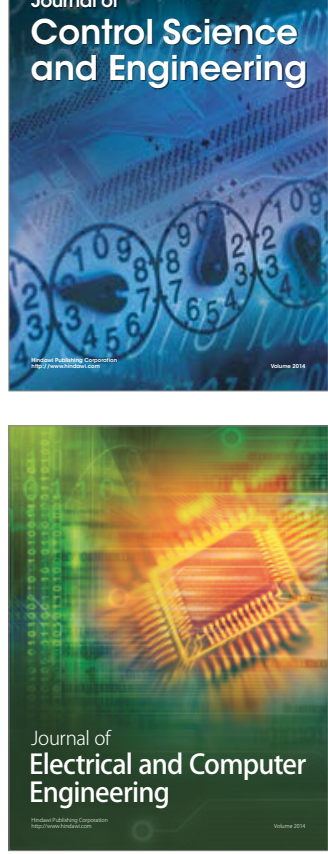

Distributed

Journal of

Control Science

and Engineering
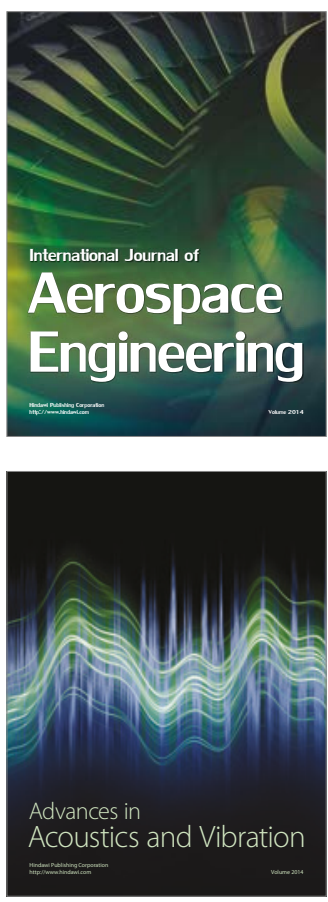

Sensor Networks 